\title{
The evaluation of ENA-78 and fibrinogen levels for the differential diagnosis of familial Mediterranean fever and acute appendicitis
}

https://doi.org/10.1515/labmed-2018-0146

Received October 1, 2018; accepted March 23, 2019; previously published online April 22, 2019

\begin{abstract}
Background: Familial Mediterranean fever (FMF) is a disorder mainly present in the Mediterranean region, characterized by abdominal attacks that mimic acute peritonitis. Therefore, FMF might be confused with other conditions presenting with acute abdominal pain, particularly acute appendicitis (AA). We aimed to evaluate whether epithelial-derived neutrophil-activating peptide-78 (ENA-78) and fibrinogen levels and white blood cell (WBC) counts could be used as potential markers in the differential diagnosis of acute FMF attacks and AA.

Methods: The study involved three groups: patients with AA who underwent surgery (group $1, n=30$ ), patients with FMF attacks (group 2, $\mathrm{n}=30$ ), and patients with FMF who were attack-free $(n=30$, controls), which included patients who were diagnosed as having FMF previously but had no attacks for a month.
\end{abstract}

\footnotetext{
*Correspondence: Mehmet Cihat Demir, MD, Department of Emergency Medicine, Sinop Atatürk State Hospital, Sinop, Turkey, Phone: +(90) 36827155 70/extension 1120, Fax: +(90) 3682714471 , E-Mail:mdcihat@gmail.com

Murat Güzel: Department of Emergency Medicine, Samsun Training and Research Hospital, University of Health Sciences, Samsun, Turkey Recep Aktimur: Department of General Surgery, Istanbul Aydın University, Istanbul, Turkey

Kadir Yıldırım: Department of General Surgery, Samsun Liv Hospital, Samsun, Turkey

Sude Hatun Aktimur: Department of Hematology, Samsun Training and Research Hospital, University of Health Sciences, Samsun, Turkey Mehmet Hakan Taşkın: Department of Medical Microbiology, Samsun Training and Research Hospital, University of Health Sciences, Samsun, Turkey Mehmet Derya Demirağ: Department of Rheumatology, Samsun Training and Research Hospital, University of Health Sciences, Samsun, Turkey
}

Results: Significant differences were detected in the ENA-78 and fibrinogen levels between group 1 and group 2. No significant difference was found in the WBC count between group 1 and group 2. It was concluded that the WBC count was more sensitive $(66 \%)$ for group 1 , and fibrinogen showed higher sensitivity (86\%) and specificity (96\%) for group 2 compared with the other parameters. Conclusions: We suggest that AA can be distinguished in patients with signs of peritonitis who were diagnosed as having FMF previously with the use of ENA-78 and fibrinogen levels; fibrinogen and ENA-78 might be helpful in discriminating between FMF attacks and AA.

Keywords: appendicitis; epithelial-derived neutrophilactivating peptide-78 (ENA-78); familial Mediterranean fever; fibrinogen; white blood cell.

\section{Introduction}

Familial Mediterranean fever (FMF) represents a prototype for episodic febrile conditions, characterized by fever attacks and painful manifestations. It is an autosomal recessive disorder that mainly affects people living in or originating from the Mediterranean basin, including Sephardic Jews, Arabs, Turks, Armenians, and others [1]. Abdominal attacks that mimic acute peritonitis are seen in $95 \%$ of patients with FMF [1]. Therefore, FMF might be confused with other conditions that present with acute abdominal pain, particularly appendicitis [2]. Initially, abdominal pain and tenderness may be localized, after which it can become more generalized. Signs of peritonitis, such as guarding, rebound tenderness, rigidity, and a dynamic ileus, are often present because abdominal pain is caused by peritoneal inflammation. These findings can be mistakenly interpreted as an acute surgical abdomen condition, leading to delayed diagnosis or unnecessary appendectomy and laparotomy [3-5]. 
FMF attacks are characterized by leukocytosis and elevated acute-phase reactants, such as erythrocyte sedimentation rate (ESR), C-reactive protein (CRP), fibrinogen, haptoglobin, C3, C4, and serum amyloid A (SAA). These inflammatory markers assist physicians in distinguishing FMF attacks from other conditions, such as viral illness, fibromyalgia, functional abdominal pain, and irritable bowel syndrome. Although most acute-phase reactants are typically normalized during the attack-free period, the elevation in these markers can persist in some cases, especially in those with severe or uncontrolled disease [6].

The widely used clinical and laboratory parameters are not specific enough to discriminate between abdominal pain caused by FMF and acute appendicitis (AA). However, diagnostic laboratory tests might decrease unnecessary surgical interventions, perforation rates, and hospitalization time. Epithelial-derived neutrophilactivating peptide-78 (ENA-78) is a member of the CXC chemokine family that is involved in leukocyte recruitment and activation in autoimmune disorders and inflammatory diseases [7-9]. It has been reported that abnormal expression of ENA-78 may be in some cases such as rheumatoid arthritis, psoriasis, autism, and bacterial meningitis. [10-14]. As a potent ELR ${ }^{+}$CXC chemokine, ENA-78 plays a role in the recruitment and activation of polymorphonuclear leukocytes (PMNLs), which are elevated in patients with infections. PMNLs are among the first cells to exit the peripheral blood and migrate to the inflammatory site [3, $10,15]$. We aimed to evaluate whether ENA-78 level, fibrinogen level, and the white blood cell (WBC) count could be used as potential parameters in the differential diagnosis of recurrent episodes of FMF and AA.

\section{Materials and methods}

This prospective study was approved by the Ethics Committee of Ondokuz Mayis University (approval ID: 2013/381). Written informed consent was obtained from all patients before participation in the study. In total, 90 patients were included in the study. The participants were divided into three groups: patients with AA who underwent surgery $(\mathrm{n}=30$, group 1), patients with FMF who presented with acute peritonitis ( $\mathrm{n}=30$, group 2), and patients with FMF who were attack-free for a month ( $n=30$, control group). Patients with acute abdomen causes such as acute pancreatitis and acute gastritis were excluded after laboratory and imaging findings. FMF was diagnosed in accordance with the Tel-Hashomer clinical criteria: major criteria: (a) recurrent fever together with serositis, (b) amyloidosis without any other susceptible agent, and (c) good response to continual treatment with colchicine, and minor criteria: (a) recurrent fever, (b) erysipelas-like erythema, and (c) positive familial background. Two major criteria or two minor criteria along with one major criterion indicate a definite diagnosis of FMF [16]. Patients with a disorder that might cause inflammation and affect the outcomes, those on non-cytokine agents that might influence the cytokine levels, patients with diabetes mellitus or metabolic syndrome, perforated appendicitis, and negative appendectomy, those with an inaccurate diagnosis of FMF, and pregnant patients were excluded. Demographic data and contact information were recorded for all patients.

\section{Biochemical analysis}

Serum samples were obtained at admission from patients with FMF attack and before the surgery from those with AA. The blood samples were centrifuged at $3500 \mathrm{rpm}$ for $10 \mathrm{~min}$ and plasma was removed and stored in a $-70{ }^{\circ} \mathrm{C}$ freezer required for analysis. On the day of the laboratory examination, the plasma samples were removed from the freezer and allowed to dissolve. Serum ENA-78 levels were quantified with a sandwich enzyme-linked immunosorbent assay (ELISA) (Human ENA-78 ELISA kits, SunRed, Catalogue No.: 201-12-0132, Shanghai, China), using the Infinite 200 PRO NanoQuant microplate reader (Tecan, Grödig, Austria) for detection. Fibrinogen and WBC counts were evaluated concurrently in the emergency laboratory. The WBC count was obtained using the Beckman Coulter LH 780 hematology analyzer (Brea, CA, USA). Fibrinogen levels were measured using a Sysmex CA-1500 automated blood coagulation analyzer (Sysmex Corporation, Kobe, Japan).

\section{Statistical analysis}

Data were analyzed using IBM SPSS version 23.0 software. The Kolmogorov-Smirnov test was used to analyze the data distribution. One-way analysis of variance (ANOVA) was used to compare the findings of the groups. Tukey's honestly significant difference (HSD) and Tamhane's T2 tests were used to perform multiple comparisons. The results are expressed as mean \pm standard deviation and frequency (percentage). Spearman's correlation test was used for correlation analysis. Receiver operating characteristic (ROC) curve analysis was used to determine the cut-off values for the fibrinogen, ENA-78 values, and WBC counts in group 1 and group 2 . p-Values $<0.05$ were considered to be statistically significant. 


\section{Results}

Of the 90 patients included in the study, 54 (60\%) were women and $36(40 \%)$ were men. No statistically significant difference was found between the sexes in terms of fibrinogen, ENA-78, and WBC counts $(p=0.283, p=0.600$, and $\mathrm{p}=0.128$, respectively). The mean ages of the participants were $28.4 \pm 10.6$ in group $1,32.3 \pm 12.6$ years in group 2 , and $31.2 \pm 10.4$ years in the controls. There was no significant difference in the mean ages of the three groups $(\mathrm{p}=0.330)$. The mean body mass index (BMI) was $24.3 \pm$ $4 \mathrm{~kg} / \mathrm{m}^{2}$ in group $1,26.4 \pm 4.1 \mathrm{~kg} / \mathrm{m}^{2}$ in group 2 , and $25.6 \pm$ $4 / \mathrm{m}^{2}$ in the controls, indicating no significant differences between the groups $(\mathrm{p}=0.081)$.

Significant differences were found in the mean fibrinogen values between the groups $(p<0.001)$. The mean fibrinogen value was $290.3 \pm 67.3 \mathrm{mg} / \mathrm{dL}$ in group 1 , $412 \pm 56.5 \mathrm{mg} / \mathrm{dL}$ in group 2 , and $182.9 \pm 43.3 \mathrm{mg} / \mathrm{dL}$ in the controls. The highest mean value was detected in group 2, and the lowest was found in the controls.

The mean ENA-78 values differed significantly between the groups $(p<0.001)$. The mean ENA-78 value was $15.7 \pm 7.3 \mathrm{pg} / \mathrm{mL}$ in group $1,36.1 \pm 23.6 \mathrm{pg} / \mathrm{mL}$ in group 2 , and $5.7 \pm 2.7 \mathrm{pg} / \mathrm{mL}$ in the controls. The highest ENA-78 value was detected in group 2 and the lowest was in the controls.
Significant differences were found in the mean WBC counts between the groups $(\mathrm{p}<0.001)$. The mean WBC count was $11.1 \pm 2.4 \mathrm{~mm}^{3}$ in group $1,11.4 \pm 2.8 \mathrm{~mm}^{3}$ in group 2, and $7.1 \pm 2.1 \mathrm{~mm}^{3}$ in the controls. There was no significant difference between groups 1 and 2, but the mean WBC count was lower among the controls compared with groups 1 and 2 (Table 1). The sensitivity, specificity, and positive and negative predictive values (PPV and NPV) were calculated for group 1 and group 2 (Tables 2 and 3). The WBC count was detected to be more sensitive (66\%) in group 1, whereas fibrinogen showed a higher sensitivity (86\%) and specificity (96\%) for group 2 compared with the other parameters. There was a weak positive relationship between WBC count and ENA-78 in group 2. In group 1, there was a strong positive relationship between WBC count and fibrinogen. There was no significant relationship between the other parameters. When each group was evaluated in itself, no statistically significant relationship was found between fibrinogen and ENA-78 (Table 4). Figures 1 and 2 show ROC curves of fibrinogen level, ENA-78 level, and WBC count in group 1 and group 2. The calculation of area under the curve (AUC) reveals that WBC count in group 1 and fibrinogen level in group 2 are more effective than the other parameters.

Table 1: Comparison of the fibrinogen and ENA-78 values and WBC counts between the three groups.

\begin{tabular}{lrrrr}
\hline & Group 1 & Group 2 & Controls & p-Value \\
\hline Fibrinogen, mg/dL & $290.3 \pm 67.3^{\mathrm{a}}$ & $412.3 \pm 56.5^{\mathrm{b}}$ & $182.9 \pm 43.3^{\mathrm{c}}$ & $<0.001$ \\
ENA-78, pg/mL & $15.7 \pm 7.3^{\mathrm{a}}$ & $36.1 \pm 23.6^{\mathrm{b}}$ & $5.7 \pm 2.7^{\mathrm{c}}$ & $<0.001$ \\
WBC, $\mathrm{mm}^{3}$ & $11.1 \pm 2.4^{\mathrm{a}}$ & $11.4 \pm 2.8^{\mathrm{a}}$ & $7.1 \pm 2.1^{\mathrm{b}}$ & $<0.001$ \\
\hline
\end{tabular}

$a, b, c$ No statistical difference was found between the groups with the same letter. Bold values were considered statistically significant $(p<0.05)$.

Table 2: The fibrinogen, ENA-78, and WBC cut-off levels and the sensitivity, specificity, PPV, and NPV for group 1.

\begin{tabular}{|c|c|c|c|c|c|c|c|c|}
\hline & Cut-off & AUC & $95 \% \mathrm{Cl}$ & Sensitivity, \% & Specificity, \% & PPV, \% & NPV, \% & p-Value \\
\hline Fibrinogen & $>263 \mathrm{mg} / \mathrm{dL}$ & 0.499 & $0.381-0.617$ & 51 & 66 & 75 & 40 & 0.983 \\
\hline ENA-78 & $>13 \mathrm{pg} / \mathrm{mL}$ & 0.521 & $0.404-0.639$ & 50 & 70 & 76 & 41 & 0.742 \\
\hline WBC & $>10,000 / \mathrm{mm}^{3}$ & 0.687 & $0.575-0.798$ & 66 & 66 & 80 & 50 & 0.004 \\
\hline
\end{tabular}

Bold values were considered statistically significant $(p<0.05)$.

Table 3: The fibrinogen, ENA-78, and WBC cut-off levels and the sensitivity, specificity, PPV, and NPV for group 2.

\begin{tabular}{|c|c|c|c|c|c|c|c|c|}
\hline & Cut-off & AUC & $95 \% \mathrm{Cl}$ & Sensitivity, \% & Specificity, \% & PPV, \% & NPV, \% & p-Value \\
\hline Fibrinogen & $>347 \mathrm{mg} / \mathrm{dL}$ & 0.958 & $0.921-0.996$ & 86 & 96 & 98 & 78 & $<0.001$ \\
\hline ENA-78 & $>19 \mathrm{pg} / \mathrm{mL}$ & 0.914 & $0.860-0.969$ & 78 & 90 & 94 & 67 & $<0.001$ \\
\hline WBC & $>10,000 / \mathrm{mm}^{3}$ & 0.712 & $0.604-0.819$ & 65 & 63 & 78 & 47 & 0.001 \\
\hline
\end{tabular}

Bold values were considered statistically significant $(p<0.05)$. 
Table 4: Correlation of fibrinogen, ENA-78, and WBC count in all groups.

\begin{tabular}{|c|c|c|}
\hline & WBC & ENA-78 \\
\hline \multicolumn{3}{|c|}{ FMF-attack } \\
\hline \multicolumn{3}{|c|}{ Fibrinogen } \\
\hline$r$ & 0.215 & 0.136 \\
\hline $\mathrm{p}$ & 0.255 & 0.474 \\
\hline \multicolumn{3}{|c|}{ ENA-78 } \\
\hline$r$ & 0.382 & \\
\hline$p$ & 0.037 & \\
\hline \multicolumn{3}{|c|}{ Appendicitis } \\
\hline \multicolumn{3}{|c|}{ Fibrinogen } \\
\hline$r$ & 0.767 & 0.230 \\
\hline $\mathrm{p}$ & $<0.001$ & 0.222 \\
\hline \multicolumn{3}{|c|}{ ENA-78 } \\
\hline$r$ & 0.189 & \\
\hline$P$ & 0.317 & \\
\hline \multicolumn{3}{|c|}{ FMF-attack free } \\
\hline \multicolumn{3}{|c|}{ Fibrinogen } \\
\hline$r$ & 0.038 & -0.056 \\
\hline $\mathrm{p}$ & 0.841 & 0.769 \\
\hline \multicolumn{3}{|c|}{ ENA-78 } \\
\hline$r$ & -0.008 & \\
\hline $\mathrm{p}$ & 0.968 & \\
\hline
\end{tabular}

Bold values were considered statistically significant $(p<0.05)$.

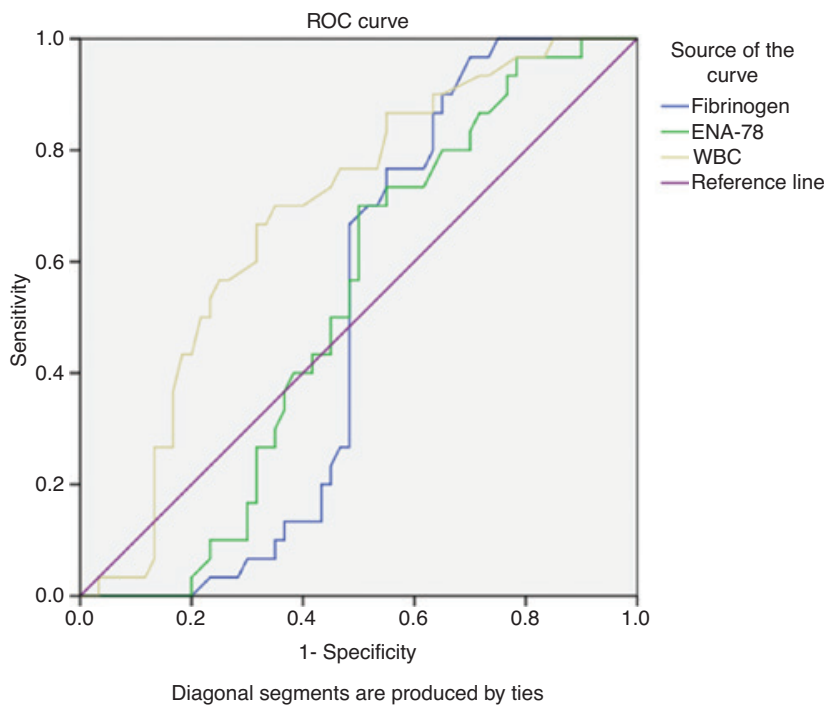

Figure 1: The sensitivity and specificity for fibrinogen and ENA-78 levels, and WBC count in group 1 according to the ROC curve.

\section{Discussion}

The frequency of appendectomy is $12-25 \%$ in the general population and $40 \%$ in patients with FMF [17]. In patients with FMF who present with acute peritonitis, laparotomy is the most commonly performed procedure due to AA [18].

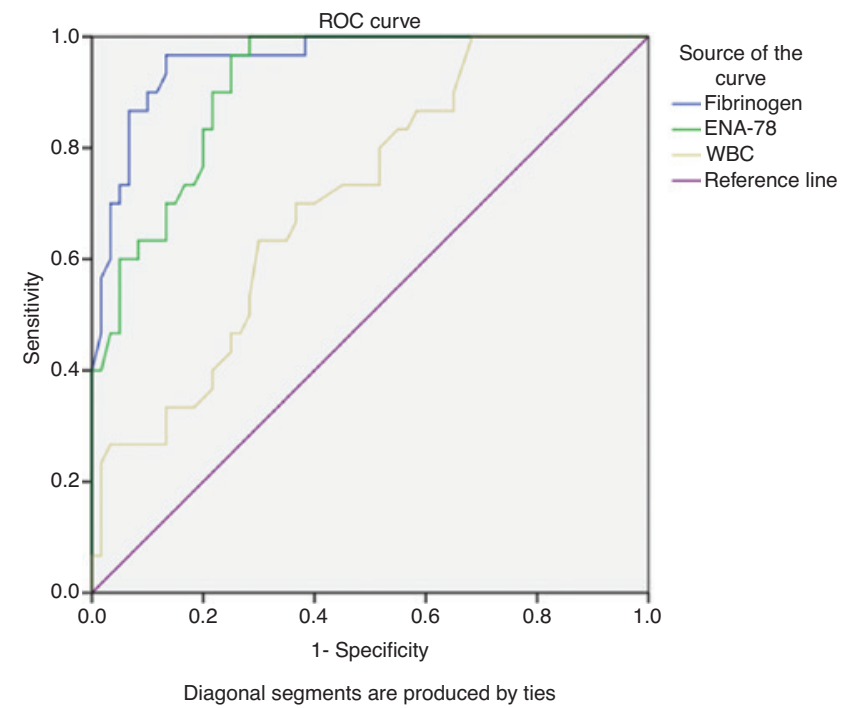

Figure 2: The sensitivity and specificity for fibrinogen and ENA-78 levels, and WBC count in group 2 according to the ROC curve.

One of the most common causes of diagnostic uncertainty in patients who present to emergency departments with abdominal pain is that a diagnosis of AA cannot be excluded in a patient with or without FMF who do not undergo an appendectomy [19]. Thus, several biochemical tests are ordered to help diagnose these patients, including WBC counts, and fibrinogen, ESR, and CRP analyses [6].

To date, many clinical findings and biochemical markers have been introduced to effectively differentiate these two conditions [20-25]; however, no single laboratory or clinical factor is able to provide discriminative outcomes. Kisacik et al. [21] stated that serum resistin and visfatin levels failed to distinguish AA from acute FMF attacks. In another study conducted by Kisacik et al. [23], procalcitonin levels were found to be significantly higher in patients with appendicitis than in patients with FMF. However, more comprehensive studies are needed before the implementation routine of procalcitonin testing can be recommended.

Kucuk et al. [25] indicated that the neutrophil-tolymphocyte (NLR) ratio might be helpful in distinguishing an acute FMF attack from AA because it is a readily available marker. Ugan et al. [24] stated that urinary $\beta_{2}$-microglobulin (U- $\beta 2 \mathrm{M}$ ) assay might be helpful in the differential diagnosis of peritonitis attacks and AA in patients with FMF on colchicine treatment. We planned this study because there is a limited number of studies on this issue and we think that the findings will lead to the use of new biomarkers. We sought to determine whether WBC counts and ENA-78 and fibrinogen would be useful in distinguishing the two diseases. 
ENA-78 is a chemokine that is a potent stimulator of neutrophils; it induces a variety of biologic responses, such as chemotaxis, enzyme release, the upregulation of surface receptors, and intracellular calcium mobilization [26-28]. The production of ENA-78 and other chemokines can result in a chemotactic gradient that has the capacity to increase the migration of granulocytes and monocytes/ macrophages from the bloodstream through the endothelium, resulting in a marked increase in chronic inflammation $[28,29]$. In rheumatoid arthritis, it was reported that local ENA-78 production occurred in the joints [30, 31]. The predominance of several chemokines in other collagen diseases, such as systemic lupus erythematosus and systemic sclerosis, has also been described [29, 32]. Thus, we aimed to evaluate whether ENA-78 level could be used as a potential parameter for use in the differential diagnosis of recurrent episodes of FMF and AA.

When we compared the ENA-78 and fibrinogen values and the WBC counts in our study, we found significant differences in ENA-78 and fibrinogen values, but not in WBC counts between group 1 and group 2 (Table 1). When the sensitivity and specificity of the evaluated parameters were assessed, the sensitivity of the WBC count was higher for group 1, whereas fibrinogen sensitivity was higher for group 2.

The WBC count is a laboratory parameter widely used in both FMF and AA in emergency departments [6]. However, in the context of current information, there are no data showing that the WBC count can be used alone to differentiate between FMF attacks and AA. In a review encompassing studies published between 2010 and 2015, Acharya et al. [33] found that the sensitivity and specificity of the WBC count were $79 \%$ (95\% confidence interval [CI]: 0.78-0.81) and 55\% (95\% CI: 0.54-0.57), respectively. Using this approach, the accuracy of a diagnosis of appendicitis can be enhanced, reducing the number of negative appendectomies performed, thereby reducing the implied adverse impact to patients and treatment costs to hospitals [34]. In our study, it was found that there were significant differences in WBC counts between the controls and the other two groups; however, this finding had no effect on discriminating between AA (group 1) and FMF attacks (group 2) (Table 1). The sensitivity and specificity of the WBC counts were $66 \%$ in the AA group and $65 \%$ in the FMF attack group (Tables 2 and 3). Although the WBC count appeared to be a marker in the diagnosis of AA, it seems that it cannot distinguish FMF attacks from AA.

In emergency departments, fibrinogen analysis is a commonly ordered test to diagnose acute FMF attacks. Fibrinogen accumulation is an expected feature in damaged tissues and inflammatory foci. In vitro studies have shown that fibrinogen can deeply alter WBC function, leading to changes in cell migration, phagocytosis, chemokine and cytokine production, degranulation, and other processes. Many of the effects of fibrinogen on leukocyte activity appear to be mediated by a specific receptor on leukocytes, the integrin receptor $\alpha \mathrm{M} \beta 2$ $[34,35]$. In this respect, studies have been performed to determine if fibrinogen may be used as a biomarker to reduce negative laparotomies in suspected AA. Menteş et al. [36] indicated that serum values of fibrinogen could be used as a new acute-phase reactant in the diagnosis of AA.

Nyuwi et al. reported that fibrinogen had a sensitivity of $88 \%$ and a specificity of $55 \%$ in the diagnosis of AA. They found that the use of fibrinogen serum levels in the diagnosis of AA might reduce the rate of negative appendectomies [37]. In our study, there were significant differences in the fibrinogen values between the groups (Table 1). In addition, the sensitivity and specificity of fibrinogen were $51 \%$ and $66 \%$ in group 1 and $86 \%$ and $96 \%$ in group 2 , respectively.

There are some limitations to our study. First, the number of participants in each group was limited; therefore, it limits the power to detect changes in markers as variable as ENA-78, fibrinogen, and WBC count. Second, it would be better to include a group of healthy volunteers with no FMF, especially in view of the baseline values for ENA-78. Third, in patients with FMF, ENA-78 and fibrinogen may increase in the presence of conditions that cause peritonitis. These biomarkers are useful in distinguishing FMF from AA if the cause of peritonitis is AA. No comparison was made with other diseases that may be the cause of peritonitis. Finally, the study could be supported by CRP, ESR, haptoglobin, C3, C4 and other acute-phase reactants. Extensive haptoglobin and complement factor measurements were beyond the scope of this study and were therefore not performed.

\section{Conclusions}

Fibrinogen and ENA-78 might be helpful in discriminating FMF attacks from AA. We recommend routinely studying ENA-78 and fibrinogen in patients with FMF who present with peritonitis in order to distinguish between an FMF attack and AA.

Author contributions: All the authors have accepted responsibility for the entire content of this submitted manuscript and approved submission. 
Research funding: None declared.

Employment or leadership: None declared.

Honorarium: None declared.

Competing interests: The funding organization(s) played no role in the study design; in the collection, analysis, and interpretation of data; in the writing of the report; or in the decision to submit the report for publication.

Compliance with ethical standards: No conflict of interest was declared by the authors.

\section{References}

1. Sohar E, Gafni J, Pras M, Heller H. Familial Mediterranean fever: a survey of 470 cases and review of the literature. Am J Med 1967;43:227-53.

2. Tunca M, Akar S, Onen F, Ozdogan H, Kasapcopur O, Yalcinkaya F, et al. Familial Mediterranean fever (FMF) in Turkey: results of a nationwide multicenter study. Medicine 2005;84:1-11.

3. Deng M, Ma T, Yan Z, Zettel KR, Scott MJ, Liao H, et al. Toll-like receptor 4 signaling on dendritic cells suppresses polymorphonuclear leukocyte CXCR2 expression and trafficking via interleukin 10 during intra-abdominal sepsis. J Infect Dis 2016;213:1280-8.

4. Haghighat M, Derakhshan A, Karamifar H. Familial Mediterranean fever. Shiraz E-Med J 2006;7:1-18.

5. Ben-Chetrit E, Levy M. Familial Mediterranean fever. Lancet 1998;351:659-64.

6. Berkun Y, Padeh S, Reichman B, Zaks N, Rabinovich E, Lidar M, et al. A single testing of serum amyloid $A$ levels as a tool for diagnosis and treatment dilemmas in Familial Mediterranean fever. Semin Arthritis Rheum 2007;37:182-8.

7. Sanz MJ, Kubes P. Neutrophil active chemokines in in vivo imaging of neutrophil trafficking. Eur J Immunol 2012;42:278-83.

8. Lira SA, Furtado GC. The biology of chemokines and their receptors. Immunol Res 2012;54:111-20.

9. Smit JJ, Lukacs NW. A closer look at chemokines and their role in asthmatic responses. Eur J Pharmacol 2006;533:277-88.

10. Zwijnenburg PJ, de Bie HM, Roord J, van der Poll T, van Furth AM. Chemotactic activity of CXCL5 in cerebrospinal fluid of children with bacterial meningitis. J Neuroimmunol 2003;145: 148-53.

11. Kawamura M, Toiyama Y, Tanaka K, Saigusa S, Okugawa Y, Hiro J, et al. CXCL5, a promoter of cell proliferation, migration and invasion, is a novel serum prognostic marker in patients with colorectal cancer. Eur J Cancer 2012;48:2244-51.

12. Sundaram K, Rao DS, Ries WL, Reddy SV. CXCL5 stimulation of RANK ligand expression in Paget's disease of bone. Lab Invest 2013;93:472-9.

13. Mostafa GA, Al-Ayadhi LY. The possible link between elevated serum levels of epithelial cell-derived neutrophil-activating peptide-78 (ENA-78/CXCL5) and autoimmunity in autistic children. Behav Brain Funct 2015;11:11.

14. Gillitzer R, Ritter U, Spandau U, Goebeler M, Bröcker EB. Differential expression of GRO- $\alpha$ and IL- 8 mRNA psoriasis: a model for neutrophil migration and accumulation in vivo. J Invest Dermatol 1996;107:778-82.
15. Rocha BC, Marques PE, Leoratti FM, Junqueira C, Pereira DB, do Valle Antonelli LR, et al. Type I interferon transcriptional signature in neutrophils and low-density granulocytes are associated with tissue damage in malaria. Cell Rep 2015;13:2829-41.

16. Livneh A, Langevitz P, Zemer D, Zaks N, Kees S, Lidar T, et al. Criteria for the diagnosis of familial Mediterranean fever. Arthritis Rheum 1997;40:1879-85.

17. Lidar M, Doron A, Kedem R, Yosepovich A, Langevitz P, Livneh A. Appendectomy in familial Mediterranean fever: clinical, genetic and pathological findings. Clin Experiment Rheumatol 2008;26:568.

18. Kasifoglu T, Cansu DÜ, Korkmaz C. Frequency of abdominal surgery in patients with familial Mediterranean fever. Int Med 2009;48:523-6.

19. Kisacik B, Karabicak I, Erol MF, Ozer S, Pehlivan Y, Onat AM, et al. Is familial Mediterranean fever (FMF) common in patients with negative appendectomy? Modern Rheumatol 2013;23: 330-3.

20. Onen F. Familial mediterranean fever. Rheumatol Int 2006;26:489-96.

21. Kisacik B, Erol MF, Yilmaz G, Yilmaz FM, Maras Y, Kalyoncu U, et al. Resistin and visfatin: are they valuable enough to be the differential diagnosis in familial Mediterranean fever with acute appendicitis? Clin Rheumatol 2012;31:225-9.

22. Shohat M, Halpern GJ. Familial Mediterranean fever - a review. Genet Med 2011;13:487.

23. Kisacik B, Kalyoncu U, Erol MF, Karadag O, Yildiz M, Akdogan A, et al. Accurate diagnosis of acute abdomen in FMF and acute appendicitis patients: how can we use procalcitonin? Clin Rheumatol 2007;26:2059-62.

24. Ugan Y, Korkmaz H, Dogru A, Koca YS, Balkarlı A, Aylak F, et al. The significance of urinary beta- 2 microglobulin level for differential diagnosis of familial Mediterranean fever and acute appendicitis. Clin Rheumatol 2016;35:1669-72.

25. Kucuk A, Erol MF, Senel S, Eroler E, Yumun HA, Uslu AU, et al. The role of neutrophil lymphocyte ratio to leverage the differential diagnosis of familial Mediterranean fever attack and acute appendicitis. Korean J Intern Med 2016;31:386-91.

26. Luster AD. Chemokines-chemotactic cytokines that mediate inflammation. N Engl J Med 1998;338:436-45.

27. Mahalingam SK. Chemokines and chemokine receptors in infectious diseases. Immunol Cell Biol 1999;77:469-75.

28. Wuyts AP, Lenaerts JP, Ben-Baruch A, Van Damme J, Wang JM. Differential usage of the CXC chemokine receptors 1 and 2 by interleukin- 8 , granulocyte chemotactic protein- 2 and epithelial-cell-derived neutrophil attractant-78. Eur J Biochem 1998;255:67-73.

29. Segerer S, Nelson PJ, Schlöndorff D. Chemokines, chemokine receptors, and renal disease: from basic science to pathophysiologic and therapeutic studies. J Am Soc Nephrol 2000;11: 152-76.

30. Halloran MM, Woods JM, Strieter RM, Szekanecz Z, Volin MV, Hosaka S, et al. The role of an epithelial neutrophil-activating peptide-78-like protein in rat adjuvant-induced arthritis. J Immunol 1999;162:7492-500.

31. Badolato R, Oppenheim JJ. Role of cytokines, acute-phase proteins, and chemokines in the progression of rheumatoid arthritis. Semin Arthritis Rheum 1996;26:526-38.

32. Egido J. Chemokines, chemokine receptors and renal disease. Kidney Int 1999;56:347-8. 
33. Acharya A, Markar SR, Ni M, Hanna GB. Biomarkers of acute appendicitis: systematic review and cost-benefit trade-off analysis. Surg Endosc 2017;31:1022-31.

34. Flick MJ, Du X, Witte DP, Jiroušková M, Soloviev DA, Busuttil SJ, et al. Leukocyte engagement of fibrin(ogen) via the integrin receptor alphaMbeta2/Mac-1 is critical for host inflammatory response in vivo. J Clin Invest 2004;113: 1596-606.
35. Tang L, Eaton JW. Fibrin(ogen) mediates acute inflammatory responses to biomaterials. J Exp Med 1993;178:2147-56.

36. Menteş O, Eryılmaz M, Harlak A, Ozturk E, Tufan T. The value of serum fibrinogen level in the diagnosis of acute appendicitis. Ulus Travma Acil Cerrahi Derg 2012;18:384-8.

37. Nyuwi KT, Singh CG, Khumukcham S, Rangaswamy R, Ezung YS, Chittvolu SR, et al. The role of serum fibrinogen level in the diagnosis of acute appendicitis. J Clin Diagn Res 2017;11:PC13-5. 